\title{
CJ-F: The Kinematics of 241 AGN
}

\author{
S.Britzen*1, R.C. Vermeulen*, G.B. Taylor ${ }^{\dagger}$, R.M. Campbell ${ }^{+}$, \\ I.W. Browne ${ }^{\diamond}$, P. Wilkinson ${ }^{\diamond}$, T.J. Pearson' and A.C.S. Readhead \\ ${ }^{*}$ NFRA, P.O. Box 2, NL-7990 AA Dwingeloo, The Netherlands; \\ ${ }^{1}$ Present address: Landessternwarte, Königstuhl, 69117 Heidelberg, \\ Germany; ${ }^{\dagger}$ NRAO, P.O. Box O, Socorro, NM 87801, USA; ${ }^{+} J I V E$, \\ P.O. Box 2, NL-7990 AA Dwingeloo, The Netherlands; ${ }^{\diamond} N R A L$, Jodrell \\ Bank, Macclesfield, Cheshire SK11 9 DL, England UK; 'CIT, \\ Department of Astronomy, 105-24, Pasadena, CA 91125, USA
}

\begin{abstract}
We present first results from the analysis of multi-epoch VLBI observations of 241 sources in the CJ-F sample (Caltech JodrellFlat-spectrum, Taylor et al. 1996). We have been obtaining $5 \mathrm{GHz}$ VLBI (global and VLBA) snapshot observations of the CJ-F sources (complete with 293 sources) since 1990 in order to create a valid database for thorough statistical tests of pc-scale jet motion in AGN. All 241 CJ-F AGN have been observed at least three times with the VLBA, which enables us to investigate jet component motions and paths. In particular, we concentrate on the analysis of those properties supposed to be essential for gamma-ray production, i.e., superluminal motion and bending.
\end{abstract}

\section{Introduction}

While detailed studies of individual objects are undoubtedly important for understanding the origin and collimation of the jets and their emission mechanisms, a full understanding will only come from study of large, well-defined samples that can be subjected to statistical analysis. Taking note of the controversy in the literature over, for instance, the physical nature of jet components, pattern and bulk speeds, and unified schemes, the next step is to improve both the amount and the quality of the observational data under consideration. To this end we are analysing the results of the CJ-F sample. By means of a detailed analysis of the sources, we plan to focus on the following source characteristics: the structural variability (here in particular the motion statistics) and the broad-band activity. Theoretical models for the production of jet emission can directly be tested for a meaningful number of sources by combining the information from VLBI radio observations and observations in other spectral regions particularly gamma-ray.

\section{Observations}

The new, almost completed VLBI survey "CJ-F" yields an unprecedented basis for a statistical analysis of jets in AGN. The CJ-F, is a complete flux-limited sample of flat-spectrum radio sources drawn from the $6 \mathrm{~cm}$ and $20 \mathrm{~cm}$ Green 
Bank Surveys (Gregory \& Condon 1991; White \& Becker 1992) with selection criteria as follows: $S(6 \mathrm{~cm}) \geq 350 \mathrm{mJy}, \alpha_{20}^{6} \geq-0.5, \delta(1950) \geq 35^{\circ}$, and $\left|b^{\mathrm{II}}\right| \geq 10^{\circ}$. We aim at obtaining at least three observing epochs, spread over roughly 4 years, to enable unambigiuous motion parameters. These observations are now $96 \%$ complete; the last epoch for a subsample of 34 sources will be obtained in December. The data reduction is $92 \%$ complete. For 241 objects, three epochs of observations have now been obtained and reduced. For 32 sources, either no redshift information is available or the source reveals no structure. For the remaining 209 objects, jet component motion can be studied.

\section{The gamma-bright subsample}

Strong gamma-ray emission and blazar properties are physically related : with 66 high-confidence blazar identifications of sources detected by EGRET (Hartman et al. 1999), it has become increasingly clear that $\mathrm{GeV}$ gamma-ray sources are preferentially radio-bright, compact-core, flat-spectrum sources, many of which have been classified as optically violent variables (OVV) or blazars. The active galaxies Mrk 421 and Mrk 501, and - at a lower level of significance - a few other AGN (some are members of the CJ-F sample) have been detected by ground-based Cherenkov telescopes at the highest-energy end of the electromagnetic spectrum accessible with current technology (TeV-regime). Superluminal motion is common to all $\gamma$-ray sources studied in sufficient detail (e.g., Barthel 1995, Bower et al. 1997, Britzen et al. 1999b, Britzen et al. 2000a). Many sources also exhibit a parsec-scale jet that is bent or is misaligned with respect to the kiloparsec-scale jet (see, e.g., Bower et al. 1997). It has been suggested that relativistic beaming may explain the observation that although all EGRETidentified AGNs are radio loud with flat spectra, not all radio-loud flat-spectrum AGNs are detectable gamma-ray sources. In order to test these hypothesis, we started an analysis of the properties of the gamma-bright blazars in the CJ-F sample - 14 CJ-F sources according to the third EGRET catalog (Hartman et al. 1999). For 11 objects, two or more VLBI observations have been performed and reduced. Here we compare results for this sample with the results obtained for the fully observed and analysed 241 objects (four sources belong to both samples).

\section{Motion}

Previous studies of motions in large samples of superluminal sources have been hampered by the fact that, taken from literature searches, they relied on smaller and/or inhomogeneously sampled data collections. Currently at least two major motion surveys are being carried out that will overcome these drawbacks: the 2-cm survey (Kellermann et al. 1998) and the current work based on the CJ-F. The apparent velocities of jet components of CJ-F sources analyzed so far show a wide range of behaviour: different components in the same source can show different velocities; components can accelerate, decelerate, merge, or split; and in some cases a stationary component can co-exist with moving components. In Fig. 1 (top) we show the $\beta_{\text {app }}$ - redshift relation for all the 209 objects. Jet component speeds have been averaged for each source and only the absolute 
values are shown (calculations use $H_{0}=65 \mathrm{~km} \mathrm{~s}^{-1} \mathrm{Mpc}^{-1}, q_{0}=0.5$ ). The tendency of higher $\beta_{\text {app }}$ at larger redshift, already visible in the earlier analysis (when most sources had only two epochs, Britzen et al.1999a), can be confirmed; it even seems to be more pronounced with the additional data epoch here. We also confirm the lower $\beta_{\text {app }}$ for BL Lac objects as compared to those for quasars or galaxies . A preliminary analysis of the average velocities of the (small) gamma-bright subsample indicates similar values as obtained for the gammaquiet objects.

\section{Curvature}

One of the important results of the analysis of the present survey is that curved jet structures seem to be a very common phenomenon in AGN. In Fig. 1 (bottom) we show the curvature observed on pc-scales by VLBI plotted against redshift. Most sources reveal jets curved by less than 50 degrees, but in some sources have curvature up to 100 degrees. In some cases quasi-oscillatory trajectories or ridge lines have been observed. Curvature is important with regard to the emission mechanisms that play a role in AGN. It definitely has to be taken into account when calculating velocities, and might significantly change the motion statistics (not yet taken into account in Fig. 1) for the fast, strongly curved objects.

A final discussion of the results has to wait until the analysis has been completed. However, some pecularities might turn out to be significant:

-significant jet curvature is visible as well in all the $\gamma$-bright sources - whether the curvature for these is higher than for the rest of the CJ-F sample (see Fig. 1 , bottom) remains to be investigated in more detail.

-multiple jet components in some sources follow a single smoothly bent path (e.g., 0219+428 and 0954+658). Also for the gamma-bright blazar PKS 0420014 , which is not in the CJ-F, a smoothly bent jet path has been observed. In this source, the jet-components also all follow the same path.

A binary black hole model for PKS 0420-014 (Britzen et al. 2000b) is capable of explaining the motion of the VLBI components as well as the observed optical flaring (see also Villata \& Raiteri 1999 for MRK 501).

Further analysis of the whole CJ-F could potentially point towards the property(ies) that discriminate gamma-loud from gamma-quiet AGN.

\section{Acknowledgements}

This work was supported by the European Commission, TMR Programme, Research Network Contract ERBFMRXCT97-0034 CERES.

\section{References}

Barthel, P.D. 1995, Highlights Astron., 10, 551

Bower, G.C., Backer, D.C., Wright, M., et al., 1997, ApJ, 484, 118 
Britzen, S., Vermeulen, R.C., Taylor, G., et al., 1999a, in ASP Conf. Ser. 159, BL Lac Phenomenon, ed. L.O. Takalo \& A. Silanpää, 431

Britzen, S., Witzel, A., Krichbaum, T.P., et al., 1999b, A\&A, 341, 418

Britzen, S., Witzel, A., Krichbaum, T.P., et al., 2000a, A\&A, 360, 65

Britzen, S., Roland, J., Laskar, J., et al., 2000b, A\&A, accepted

Gregory, P.C., \& Condon, J.J. 1991, ApJS, 75, 1011

Hartman, R.C., Bertsch, D.L., Bloom, S.D., et al., 1999, ApJS, 123, 79

Kellermann, K.I., Vermeulen, R.C., Zensus, J.A., Cohen, M.H. 1998, AJ, 115, 1295

Taylor, G.B., Vermeulen, R.C., Readhead, A.C.S., et al., 1996, ApJS, 107, 37

Villata, M. \& Raiteri, C. 1999, A\&A, 347, 30

White, R.L., \& Becker, R.H. 1992, ApJS, 79, 331
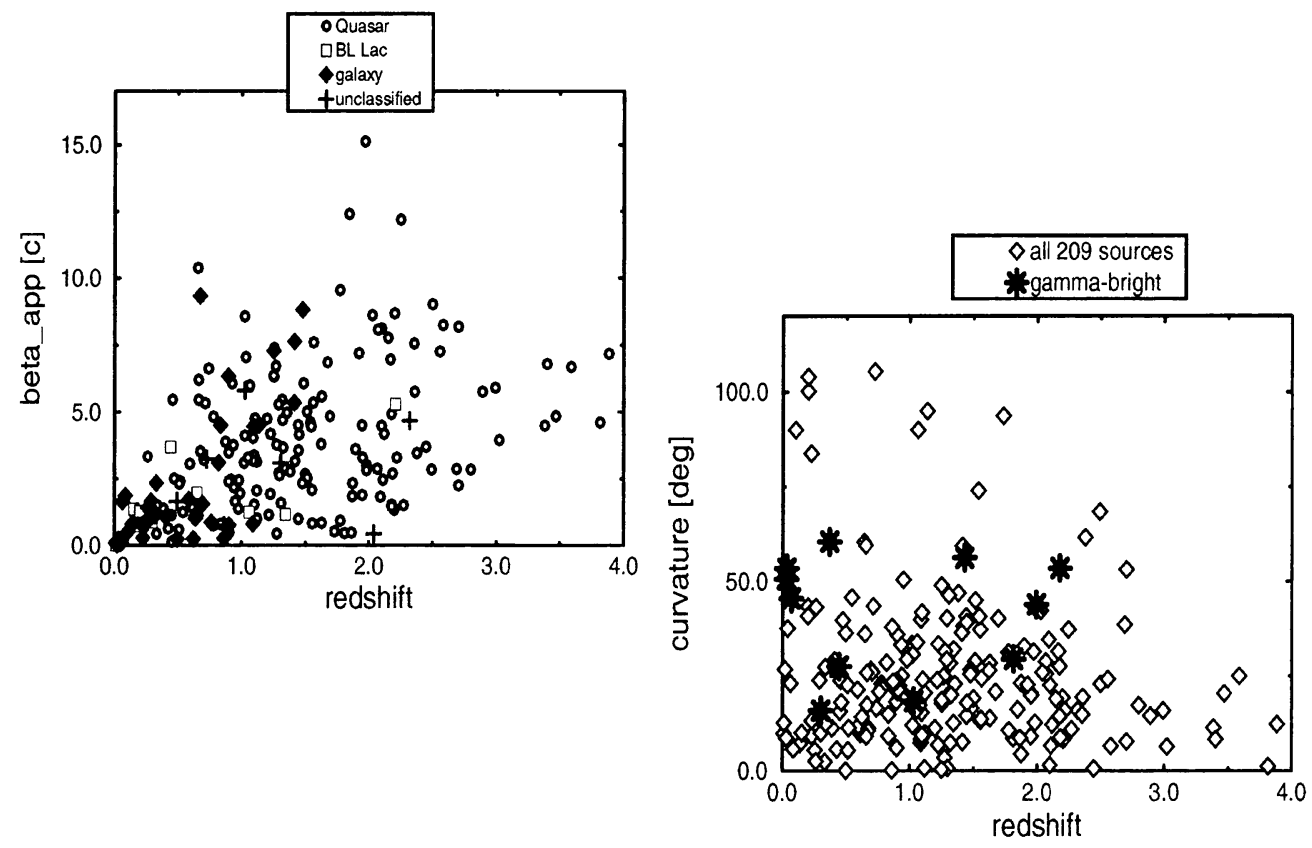

Figure 1. The figure on the left shows beta $a_{\text {app }}$ (averaged over each jet) versus redshift for the 209 sources with at least three epochs of observation. The caption introduces the symbols used for different classes of object. The figure on the right presents the relation between the curvature observed on VLBI scales and the redshift, both for the 209 gamma-quiet objects (diamonds) and the 11 gamma-bright objects (stars). 